\title{
PARA-AMINOSALICYLIC ACID IN THE TREATMENT OF TUBERCULOSIS
}

\author{
By James A. O’Connor, B.Sc., M.D., D.C.H.
}

Although it is only two and a half years ago since the first report was published on the antitubercular activity of para-aminosalicylic acid (PAS), there is already quite a considerable literature on the subject. Much of it is, however, fragmentary and it has appeared in a wide variety of journals, but apart from the early publications of the Swedish workers it has all been written in English. Many of these papers deal with only a limited aspect of the subject and in some respects experimental reports have been conflicting. This has been due mainly to scarcity of the drug in the past and to varying degrees of purity of the materials available for the earlier experiments. Now that supplies are becoming more freely available and a more satisfactory product is being offered by the various manufacturers, there is likely to be greater interest in the drug and a greater amount of research work undertaken by clinicians and others concerned with the chemotherapy of tuberculosis. The purpose of this paper is therefore to review the work that has already been done, to summarize our present knowledge of the drug and to give some indication of the work that is ahead. In preparing this paper reference has been made to every previous publication of any importance whatsoever, and whose existence it has been possible to trace.

\section{History and Development of PAS}

The original work which laid the foundations for the discovery of PAS was carried out by Bernheim (1940, 1941a, 194I b) who showed that when small amounts of sodium salicylate (sodium orthohydroxy benzoate) are added to a phosphate buffer solution of $\mathrm{pH} 6.7$ containing a suspension of tubercle bacilli the rate of oxygen consumption by the organisms is more than doubled. He also showed that sodium benzoate itself produces a similar but slighter effect, while the para- and meta-hydroxy benzoates and other salicylate compounds such as aspirin and methyl salicylate have no such action. Quantitative experiments revealed that the sodium salicylate was being oxidized as a substrate with evolution of $\mathrm{CO}_{2}$, and Bernheim himself suggested that although salicylic acid may not be a normal metabolite for the tubercle bacillus, substances of similar chemical configuration may be important. Indeed, the fact that sodium para amino benzoate has been shown to be an essential metabolite for certain streptococci and other organisms, and the observations of Bernheim described above, may indicate that substituted benzoates, of which salicylate is one, possibly play a fundamental part in general bacterial metabolism.

This work of Bernheim was subsequently confirmed and extended by Lehman (1946a) who showed that only pathogenic strains of tubercle bacilli manifest an increased oxygen consumption in the presence of salicylates and benzoates, whereas the non-pathogenic strains are not so affected, and he suggested that this test might be a useful means of distinguishing pathogenic from non-pathogenic strains. Lehman concluded from these experiments that only pathogenic tubercle bacilli must contain an enzyme or some associated factor, evidently respiratory in nature, and capable of being stimulated by salicylic acid. Since it may be assumed $a$ priori, according to Lehman, that the pathogenic properties of a bacillus must depend upon its containing certain enzyme systems, it was very suggestive that the salicylates might be exerting a direct effect upon the pathogenicity of the tubercle bacillus.

Following upon these observations Lehman (1946b, 1946c) examined a series of compounds, exceeding 60 in number, of similar chemical configurations to salicylic acid in the hope that one of them might prove capable of entering into substrate competition in a manner analogous to the sulphonamides competing with para-aminobenzoic acid (PAB). Of all the compounds tested, 4-amino-2-hydroxy-benzoic acid, or para-aminosalicylic acid (PAS) was by far the most active. So specific is this particular configuration that any movement of the amino or hydroxyl groups to other positions, or the alteration of the carboxyl group in any way, results in considerable reduction or even complete loss of anti-tubercular activity.

\section{Chemistry and Properties of PAS}

The synthesis of PAS, especially on a commercial scale, has proved to be a matter of con- 
siderable difficulty. The two methods of manufacture most commonly employed are based upon those described by Kondo and Nakajima (1922) and by Spring and others (1948), and in detail, with an account of its physical and chemical properties, by Rosdahl (1948b).

Pure PAS occurs as a white microcrystalline powder sparingly soluble in water (less than 0.2 per cent. at $20^{\circ} \mathrm{C}$.). The compound is not at all a stable one and it readily decarboxylates with formation of meta-aminophenol, particularly if moisture is present. In solution the acid decarboxylates very rapidly at temperatures above $80^{\circ} \mathrm{C}$. The melting point of PAS has been variously recorded as $138-151^{\circ} \mathrm{C}$. with decomposition, the value assigned being dependent to a great extent on the method of determination employed, the rate of heating the water bath, the temperature of immersion and similar variable factors. It is obvious, therefore, that the melting point cannot be regarded as a useful criterion of purity. The iso-electric point of PAS is at $\mathrm{pH}_{4}$.

The sodium salt occurs as the double hydrate in the form of glistening white microcrystalline needles. It is freely soluble in water to give a clear almost colourless solution, and concentrations of 25 per cent. or more may be prepared. Solutions prepared from the pure crystalline sodium salt are relatively stable and withstand temperatures in the region of $100^{\circ} \mathrm{C}$. (Seymour, 1948 ; Venkataraman and others, 1948). Hitherto the crystalline sodium salt has not been available in any quantity on account of the difficulty and cost of manufacture, and it has been necessary to prepare solutions as required from the free acid and a suitable alkali.

Whittet (1947) described a method of preparing such solutions from PAS and caustic soda and their subsequent sterilization by autoclaving. It was later pointed out by O'Connor (1948a) that only mild alkalis such as sodium bicarbonate or even carbonate should be used for this purpose if a clear solution is to be obtained. The addition of strong alkali to a suspension of PAS, even if very carefully done, produces at least a transient excess of alkalinity round the individual PAS particles, and leads to some disruption of the compound and instability of the resulting solution. Moreover, the use of strong alkalis can, under proper conditions, lead also to neutralization of the hydroxyl as well as the carboxyl radicle with the formation of a double salt, and can facilitate the formation of phenates and of hydroxylamino compounds. All sodium salt solutions prepared from the free acid are apparently capable of absorbing oxygen rapidly from the air after the manner of pyrogallic acid and of becoming gradually darker, even to reddishblack and opaque. This process is greatly accelerated by heating or by definite alkalinity of the solution. As decomposition takes place the solutions in any case become increasingly alkaline -a process which proceeds with greater rapidity once the neutral point is passed.

It is therefore necessary where sodium salt solutions must be prepared from the free acid that this should be done only a short time before use by adding slightly less than the molecular equivalent of sodium bicarbonate to an aqueous suspension of PAS and stopping the neutralization while some of the PAS particles are still undissolved, i.e. while the reaction of the solution is somewhere about $\mathrm{pH}$ 6.6-6.8. In this way deterioration of the solution may be considerably delayed. The addition of so-called stabilizers such as sulphites, which really act as bleaching agents, appears to mask discoloration and deterioration rather than prevent it.

There is now general agreement that sodium salt solutions prepared by the above method for parenteral use should be sterilized by filtration and not by heating (O'Connor, 1948a ; Seymour and McAnally, Whittet, 1948), otherwise decarboxylation takes place, a reaction which is not reversible under autoclaving conditions (Davis, 1948). So far as is known, no satisfactory method has been devised for storing these sterile filtered solutions without some further decarboxylation and deterioration. The true importance of the alterations that take place on keeping is not yet known. One is not concerned so much by the slight loss of activity due to degeneration of minute amounts of PAS into inactive compounds, but rather by the possibility that some of these degeneration products may prove to be very toxic when administered intravenously or intrathecally. It must also be remembered that small traces of impurities may be of little import where total dosage is small, but as PAS is employed in large doses over prolonged periods the cumulative effect might become a serious one.

Before leaving the chemical considerations of PAS reference must be made to the chemical estimation and assay of the material. Davis (1948) has described several methods of assay for purity of the drug, including the alkaline titration of the free acid with caustic soda, which does seem open to criticism on theoretical grounds. Though several workers have made mention of PAS blood levels achieved during therapy they do not describe in any detail the methods of estimation employed. Each laboratory appears to use a different technique with widely differing results. The adoption of a standard technique that will give comparable results is a matter of, considerable importance. McClosky and others used a modification of the Bratton and Marshall (1939) 
technique and these workers are of the opinion that diazotization of PAS can give erratic results unless due precautions are taken. A method of estimating PAS in amounts as small as I-I 5 micro gm. per $4 \mathrm{ml}$. specimen has been described by Tennant and Leland (r943). They claim that even for these low concentrations the method is accurate within \pm 5 per cent. and that it is applicable to blood filtrates and urine dilutions. Reference to the estimation of PAS by two different methods, and to the assay of some new derivatives, has also been made by Rosdahl (r 948a).

\section{Bacteriological ; Experiments in vitro}

The work of Lehman (1946c) showed that PAS acts bacteriostatically and that it does not inhibit organismal growth by bacteriolysis or other mechanism. He also confirmed a previously reported paradox that although salicylic and benzoic acids stimulate respiration of the tubercle bacillus they have also a fairly strong inhibitory effect upon its proliferation. Moreover, he showed that PAS by itself has, if anything, a stimulating effect upon the tubercle bacillus, an unexpected finding in view of the fact that PAS was devised on the theoretical basis of its entering into substrate competition with salicylic acid or substances of similar configuration. In combination with salicylic acid in the proportions of $I 0: I$ an inhibitory effect was occasionally observed but only after $30-40$ hours and it never exceeded 30 per cent. inhibition.

Sievers (1946) subsequently investigated the effect of PAS in vitro on 19 species (37 strains) of non-acid fact bacteria, both pathogenic and nonpathogenic. The test organisms included streptococci of the haemolytic, non-haemolytic and viridans varieties, staphlococcus aureus and albus, pneumococci, salmonellae, B. paratyphosus, B. coli, B. anthracis and C. diphtheriae. In no instance was there evidence of bacteriostasis at concentrations of less than $1.25-2.5$ per cent. PAS, and even at these concentrations only 13 strains of organisms had their growth completed inhibited. On the other hand, the growth of tubercle bacilli was inhibited by $10^{-5}$ molal concentrations of PAS, a figure previously -reported by Lehman (1946c).

The tuberculostatic activity of the drug was further confirmed by Youmans (1946) who checked its potency in vitro against 12 virulent strains of human type tubercle bacilli, including strains resistant to streptomycin, and reported similar results. In a subsequent paper Youmans and his co-workers (1947) further reported that of 17 strains of human type tubercle bacilli tested in vitro all were approximately equally sensitive to PAS and that the activity of the drug was not markedly affected by the presence of Io per cent. bovine plasma. No significant difference in activity was noted as between streptomycin sensitive and resistant strains, and the one strain of bovine type bacillus tested appeared to be just as susceptible as the human ones. The bacteriostatic activity of PAS was also shown by these workers to be approximately of the same order as streptomycin, since in most cases less than $\mathrm{r} \mu \mathrm{g}$. per ml. inhibited growth. As with the sulphonamides and sulphones the bacteriostatic activity of PAS is markedly influenced by the number of organisms added to the test medium, and it is apparent that for comparative results in research work the inoculum size must be standardized. It was also noted by these workers that the presence of $0.1 \mathrm{mg}$. of para-aminobenzoic acid per $100 \mathrm{ml}$. of medium reduced the bacteriostatic activity of PAS to one-sixteenth.

Duca and others (1948) also investigated the activity of PAS and found it to be the most potent, both in vitro and in vivo, among $19 \mathrm{com}^{-}$ pounds exhibiting anti-tubercular properties.

\section{Animal Experiments}

Lehman (1946c) tested the effect of PAS ro per cent. solution on rabbits by intramuscular, intravenous and oral routes, and showed that when injected intramuscularly the drug is rapidly excreted or destroyed. The material that he was using proved, however, to be much too toxic for guinea pigs, most of which died after five days treatment in which they received 5 per cent. of neutralized PAS in the diet. Death was accompanied by signs suggestive of B-complex avitaminosis. Rats, on the other hand, survived and produced normal litters after weeks of the same diet. The omentum and lymphatic glands of tuberculous infected guinea pigs treated with PAS for only five days weighed only half as much as similarly infected but untreated controls. Lehman also noted that animals which had been inoculated intraperitoneally with heat-killed tubercle bacilli also displayed distinct though less pronounced omental and lymphatic gland reactions, similar to those inoculated with virulent organisms, and that these reactions were also slightly lessened by the administration of PAS.

Youmans (1946) confirmed that PAS was at least moderately effective in suppressing tuberculous infections in mice. Youmans and co-workers (1947) later reported that the addition of 2 per cent. in the diet of a series of mice permitted an average survival time of 27.9 days in an experiment designed to last for $\mathbf{2 8}$ days, whereas the average survival time of the controls was only 20.2 days. The addition of 4 per cent. PAS to the diet resulted in a survival time of only 14.4 days, in- 
dicating that the dosage was too high and too toxic. With I per cent. of PAS added to the diet all the animals survived the full span of the experiment, but the suppression of the tubercular reaction was not as marked as when 2 per cent. was employed; the longer survival time with I per cent of PAS in the diet indicates that 2 per cent. is probably still in the toxic range of dosage. These findings are somewhat at variance with those of Lehman whom these workers state as communicating to them personally that mice tolerate well the addition of 5 per cent. PAS to the diet.

Feldman and others (1947) were able to report the survival of guinea pigs for 161 days continuous administration of 4 per cent. PAS in their food, again contrary to the findings of Lehman, who expressed the belief that PAS was too toxic for guinea pigs and that it caused death within one to two weeks. Feldman, was however, definitely impressed by the favourable effect of the drug on animals infected with tubercle bacilli even as long as six weeks after the beginning of treatment.

The work of Feldman and his colleagues has been severely criticized by McClosky and others (1948) on the grounds that their daily dose was an estimated average rather than a definite one. These workers are of the opinion that PAS has little therapeutic activity in rabbits infected with bovine strains or guinea pigs infected with human strains of tubercle bacilli. They do, however, report as a result of their experiments that the substance is relatively non-toxic, is readily absorbed and excreted, 80-90 per cent. of the drug appearing in the urine in a conjugated, probably acetylated form, the remainder being as the free acid.

Sievers (1946) showed that the administration of PAS did not modify the tuberculin reaction in guinea pigs and that it did not protect mice injected with pneumococci or with tetanus toxin.

Venkataraman and others (1948) investigated the metabolism of the drug in rabbits and report that the drug is excreted rapidly mainly in the acetylated form but also as the free acid. These workers provide much experimental evidence on 1 blood levels achieved, and on absorption and excretary rates.

\section{Glinical Trials}

The earliest clinical reports were by Lehman (1946b), who found that tuberculous abscesses following thoracoplasty showed healing after several months of daily local injections of a ro per cent. neutral solution of PAS, even though the abscess cavities had remained unchanged for 3-6 months before treatment. He also reported good results from the oral administration of the drug in daily doses of IO-1 $5 \mathrm{gm}$. in courses of eight days each, and repeated at eight day intervals. The fall in the patients' body temperatures was prompt and marked, temporary or permanent, and coincided with periods of treatment. This temperature drop was accompanied by improvement in the general condition, a gain in appetite and weight, an increase in red blood cells and haemoglobin, and a decrease in the E.S.R. No symptoms of salicism such as sweats or tinnitus were observed and Lehman therefore considered that the drop in body temperature could not be due to a salicylate effect.

Vallentin (1946) reported on a series of 75 cases with variable results. In favourable cases he observed the same response to the drug as was described by Lehman, and noted in addition the disappearance of tubercle bacilli from the sputum and improvement in X-ray appearances of the lungs. Relapses were frequent, however, when treatment was suspended and the greatest successes were obtained in cases of exudative pulmonary tuberculosis. Ten cases of tuberculous empyema treated by local injection of 5-10 per cent. solutions were not greatly benefited, whereas the instillation on alternate days of a PAS solution into postoperative extra-pleural tuberculous cavities produced healing in four cases and improvement in the remaining three. The drug was without effect in six cases of miliary tuberculosis or tuberculous meningitis. Toxic symptoms reported by Vallentin were kidney irritation with slight albuminuria, gastrointestinal upset and occasional troublesome diarrhoea. Similar results were also reported by Alin and Diffs (1947).

In Britain the first clinical trials with PAS were reported by Dempsey and Logg (1947) who treated a total of i9 patients. Six cases of pulmonary disease received the drug orally in daily doses of 1 4-20 gm., either in the form of the acid or its sodium salt. In these patients the drug exerted a beneficial effect, but the authors did not regard their results as conclusive since therapy had been intermittent on account of scarcity and irregularity in supplies of the material. They were able to report, however, that the temperature and E.S.R. fell rapidly a few days after the beginning of treatment and that over the space of a few weeks there was a fall in pulse rate and an increase in weight, vital capacity and haemoglobin. The amount of sputum decreased and the number of tubercle bacilli present in it were reduced, while the morphology of the organism changed, first becoming beaded and striated, and then being replaced after a time by acid fast granules. The patients' general condition and the radiological appearances of the lungs improved, particularly striking being the reduction in size of the lung cavities. 
Dempsey and Logg also reported one case of confirmed renal tuberculosis which cleared up completely with II weeks treatment and which had not relapsed at the time of publishing their results six months later. A single case of tuberculous meningitis complicating a pulmonary lesion was treated by oral administration of the drug and two intrathecal injections of $0.5 \mathrm{gm}$. of PAS in 5 per cent. solution with some relief of meningitic symptoms but without preserving the patient's life. The drug was also without much effect in a case of tuberculous enteritis, though its administration did reduce the diarrhoea to one semi-solid motion daily and alleviated pain. Nine cases of tuberculous empyema were also treated by intrapleural instillation of $1.5-3 \mathrm{gm}$. of PAS weekly in Io per cent. solution and some benefit was reported in several cases. Toxic symptoms recorded by these workers included nausea and vomiting though these effects were rather lessened when the sodium salt was administered instead of the free acid.

Erdei (1948, a, b) subsequently reported on five patients treated over a period of 60 days and one patient treated for four weeks with PAS administered orally in the form of a freshly prepared sodium salt solution. All six patients were given $12 \mathrm{gm}$. daily in divided doses at three-hourly intervals. The most striking effect of the drug was the very great improvement in the patients' general condition that occurred about the third day, especially in toxic, anorexic and apathetic patients. This worker also noted the considerable fall in body temperature and E.S.R., a reduction in cough, cessation of night sweats, a gain in weight and appetite, a diminution in the amount of sputum produced, and a very great reduction in the number of tubercle bacilli present in the sputum where the organisms soon became granular and tended to clump. Radiological changes were well marked in that considerable hardening and retraction of the apical lesions took place, the size of the lung cavities diminished and the areas of soft mottling cleared up. Previous to treatment with PAS these patients had received a course of 6-9 mega units of penicillin without any beneficial effect. Cultures of sputum after three weeks treatment of PAS showed no growth of tubercle bacilli after six weeks incubation, and cultures containing added para-amino benzoic acid to antagonize any PAS present in the inoculum similarly remained sterile. With daily dosage of $12 \mathrm{gm}$. blood levels of 2-5 $\mathrm{mgm}$. were achieved, but the method of their determination is not described. The urine was loaded with salicylates which were also present in the faeces in considerable amounts when the free acid was given orally, but only in faint traces when solutions of the sodium salt were employed. During treatment these patients all developed a moderate leucocytosis of $12,000-14,000$ per c.mm. with a relative increase in polymorphs, a shift to the left and a high proportion of monocytes (up to 16 per cent.). No toxic symptoms of any kind were observed in this series. This worker believes, however, that dosage should be much higher.

Way and others (1948) have reported that the oral administration of $4 \mathrm{gm}$. of PAS causes the blood level in human subjects to rise rapidly to ro mgm. per cent. and then to fall away quickly again, with 90 per cent. of the PAS being easily recoverable from the urine. They also state that the highest tissue concentrations are achieved in the kidney and next in the lungs and liver.

Ragaz (1948) reports improvement in 60 to 70 per cent. of pulmonary cases treated with Io to 15 gm. PAS daily during alternate weeks, treatment extending over several months. One renal case became bacteriologically negative in two weeks and free of symptoms after I I weeks' treatment.

\section{Mode of Action of PAS}

Although PAS was originally conceived as a material capable of entering into substrate competition with salicylic acid or metabolites of similar structure, it has been proved in actual practice that the action of PAS is far from being such a simple one. The true manner in which the drug exerts its effect is as yet far from being properly understood.

Lehman (1946c) suggests that PAS, like benzoates and salicylates, inhibits the deamination of amino acids during cellular metabolism and that PAS therefore owes its anti-tubercular activity to its capacity for inducing a disturbance in the protein metabolism of the tubercle bacillus. His earliest paper (1946a) did imply, however, that the activity of PAS was due to an anti-salicylate effect.

Youmans and his colleagues (1947) reported that the bacteriostatic activity of PAS was not influenced by the presence of sodium salicylate in the culture medium, even in concentrations between 0.1 and $1.0 \mathrm{mgm}$. per Ioo $\mathrm{ml}$., and they quote Lehman as having also reported in a personal communication that PAS did not interfere with the stimulation of bacillary respiration by salicylates. These workers are therefore inclined to the view that the drug has an action on the tissues of the host, : changing their reaction from necrotic-exudative to fibrotic-proliferative, but that it must at the same time exert a depressive action on the tubercle bacilli themselves, the latter effect being probably the greater one in view of the well established in vitro effectiveness of the drug.

Erdei (1948) is of the opinion that the drug's 
effect is a complex one, and cites the rapid reduction in the tubercle bacilli content of the sputum, together with the morphological changes produced in the organisms, as confirmatory evidence of the anti-bacterial activity of the drug that can be demonstrated in vitro. On the other hand, he regards the general clinical improvement of the patient, the anti-pyretic effect, and the stimulating action on the white corpuscles as indicating an action of the drug on the host. The radiological changes reported by Vallentin, Dempsey and Logg, and by Erdei may also be regarded as evidence that PAS promotes fibrosis in the host.

It is worth while, however, to re-scrutinize certain facts recorded by Lehman and to consider the inferences that may be drawn from them. First to be noted is his statement that pathogenic strains of tubercle bacilli must $a$ priori owe their pathogenicity to an enzyme system which is not present in non-pathogenic strains. $\mathrm{He}$ then draws attention to the fact that pathogenic strains contain an enzyme system capable of being stimulated by salicylates but that non-pathogenic strains contain no such enzyme. It is tempting to infer that the salicylate-stimulable enzyme is identical with the one conferring pathogenicity, and that PAS acts directly on this enzyme system. Yet Lehman also notes that PAS inhibits growth in vitro of the non-pathogenic B.C.G. (which contains no salicylate stimulable enzyme) equally as well as it inhibits growth of pathogenic strains.

The experimental work of Lehman (1946c), confirmed later by Feldman and others (1947), showed that the omentum of animals inoculated intraperitoneally with virulent tubercle bacilli became greatly infiltrated, thickened and heavier, and that the amount of infiltration was considerably less in inoculated animals treated with PAS. Lehman also reported that where heat-killed tubercle bacilli were used as the inoculum, the animals displayed a similar distinct though less pronounced omental infiltration (as judged by comparative weights) and that in this instance too the omental reaction was slightly reduced by administration of PAS. Unfortunately Lehman did not describe the various omental reactions in detail, nor indicate whether the infiltration from heat-treated bacteria - was exudative or fibrotic. Such an observation might have been of the greatest importance if it could have shown that virulent bacteria tend to produce an exudative and necrotic reaction in the host's tissue because of the enzymes they contain, and that apathogenic varieties or those whose enzymes have for the most part been destroyed by heating tend to cause irritative and fibrotic changes. But it has been proved that PAS does not enter into substrate competition; and yet it does inhibit the growth in vitro of B.C.G. which contains neither the pathogen enzyme nor the salicylate enzyme. It is therefore apparent that PAS does not exert its beneficial effect in cases of tuberculosis by neutralizing the bacterial enzyme systems directly, and that it must interfere with the action of the pathogen enzyme by interfering with its production. If PAS acts directly on the metabolism of the tubercle bacillus in the manner suggested by Lehman then such a disturbance of metabolism would cause bacteriostasis (loss of power of reproduction) in all types of tubercle bacilli whether they be pathogenic or not, and incidentally a diminution in the amount of pathogen enzyme in the virulent strains, thereby bringing about a change of tissue reaction in the host. The confirmation of a different type of tissue reaction by virulent and non-virulent bacteria would add very great support to Lehman's theory on the mode of action of the drug and would provide a single explanation for both the in vitro and in vivo activity of the compound, and also for its tendency to cause healing by fibrosis as expressed or implied in the observations of Vallentin, Youmans and others (1947), Dempsey and Logg, and of Erdei. The same theory would also account for the normal change of the tubercular reaction from exudative to fibrotic as healing takes place, as the patient's resistance increases, and the infecting bacillus becomes attenuated by antibody formation with loss of its capacity for producing the pathogen enzyme. If some such theory should be established then the effect of salicylates and benzoates upon the respiration of the tubercle bacillus becomes merely an interesting phenomenon, possibly useful for identifying the presence of an enzyme system that may be identical with the one conferring pathogenicity, but which has fundamentally nothing to do with the action of PAS.

\section{Discussion : Future Work with PAS}

While the anti-tubercular activity of PAS is now well established much remains to be learned about the best methods of using the drug and about its limitations. Unpublished reports so far inaicate, for example, that its local injection into pleural effusions quickly renders the fluid sterile but does not greatly influence the rate of its re-absorption. This is not surprising in view of the fact that the principal lesion is sub-pleural and that this could best be attacked by administering the drug orally. The ideal method is probably a combination of local injection and systemic administration. Similar remarks apply too in the case of tuberculous empyema, where reports on the whole have not been too favourable ; these remarks do not however apply so much to localized tuberculous abscesses when the primary infection has already been dealt with by other means (Vallentin). Like- 
wise in tuberculous meningitis favourable results could only be expected from a combination of systemic and intrathecal dosage and not from the latter alone.

The effectiveness or otherwise of PAS in bone and joint tuberculosis still remains to be investi, gated as dòes its clinical use in combination with streptomycin, though animal experiments have indicated that the effect of the two drugs together is no more than additive, and no evidence of synergistic action has been forthcoming (McClosky and other, 1948). PAS remains yet to be tried on dermatological conditions such as lupus where the drug may need to be administered orally and by local infiltration, and in suitable lung cases or in cases of tuberculous laryngitis some beneficial effect may be anticipated from the use of PAS sprays containing aerosols. Where local anaesthetics are to be employed along with PAS they must be chosen with care since many of these compounds are derivatives of para-aminobenzoic acid and may conceivably exert a neutralizing effect upon the drug. There is also some evidence that PAS promotes phagocytosis of acid-fast bacilli and the drug may be expected to have a marked effect in leprosy as did the other anti-tubercular compounds such as the sulphones (O'Connor, r948b). Owing to scarcity of supplies little if any work has been done in this field, in Britain at any rate.

Drug fastness has not so far been reported but this possibility requires early investigation as does its effect upon the tuberculin reactions of patients treated with it. The compound may prove particularly valuable in the treatment of early and ambulant cases awaiting admission to sanatoria.

With the use of ever purer compounds toxic phenomena have tended to disappear. One possibility not so far mentioned by workers in this field and one that must be borne in mind, especially as higher dosage becomes the rule, is the possible effect of PAS on the blood-prothrombin level. It has been reported by Link and his coworkers (1943) that salicylates in large doses precipitate a hypoprothrombinaemia and spontaneous haemorrhages in rats, particularly where the diet is deficient in vitamin $K$. This observation has been confirmed by the clinical experiences of Barnett and others (1942) and by Lindsay (1937), Troll and Menten (1945) and Meyer and Howard (1943). The production of haemorrhagic states from the administration of salicylates has also been reported by Hurst and Lintott (1939), Honigsberger (1943), Hurst (1943), and by Ashworth and McKemie (1944) though its connexion with an induced hypoprothrombinaemia was not always appreciated. The work of Fashena and Walker (1944), Shapiro (1944), and of Meyer and
Howard (1943) indicates that this hypoprothrombinaemic effect of the salicylates may be avoided by simultaneous administration of vitamin $\mathrm{K}$ or its analogues. Moreover, Pelner (1942) has shown that salicylates have an affinity for ascorbic acid, and it has been noted by Daniels and Everson (1936) and by Keith and Hickmans (1938) that the administration of salicylates greatly accelerates the excretion of ascorbic acid by the body. Whether or not PAS produces these same effects as the other salicylates, though possibly in less marked degree, remains yet to be discovered. Until this is known repeated checks on the blood-prothrombin levels and ascorbic acid excretion rates of all patients undergoing treatment with PAS should be made, and meanwhile the possibility of inducing a haemorrhagic state from administration of PAS must be borne in mind, especially when treating cases with any tendency to haemoptysis.

Efforts must also be directed to the finding of some means of securing prolonged absorption from parenterally administered PAS and of achieving higher blood concentrations over longer periods. In view of the many months over which PAS treatment must extend it is not likely that the simultaneous administration of another drug to secure renal blockage of PAS will prove desirable, even if it should be possible. Of much more importance is the study of the fundamental mode of action of PAS, for a proper appreciation of this may lead to a better understanding of the mechanisms involved and to the possible synthesis of even more effective compounds.

\section{BIBLIOGRAPHY}

ASHWORTH, C. T., and MCKEMIE, J. F. (1944), F. Amer. Med. As., 126, 806 .

ALIN, K., and DIFFS, H. (1947), Nord. Med., 33, 151 .

BARNETT, H. L., and others (1942), F. Pediat., $21,214$.

BERNHEIM, F. (1940), Science, 92, 204.

BERNHEIM, F. (1941a), $\mathcal{F}$. Bact., 41, 387.

BERNHEIM, F. (194Ib), F. Pharmacol., 73, 78.

BRATTON, A. C., and MARSHALL, E. K. (1939), F. Biol Chem., 128, 537.

DANIELS, A. L., and EVERSON, G. J. (1936), Proc. Soc. Exp. Biol. Med., 35, 20.

DAVIS H. (1948), Month. Bull., Ministry of Health, 7, 109.

DEMPSEY, T. G., and LOGG, M. H. (1947), Lancet, 2, 87 I.

DUCA, C. J., WILLIAMS, R. D., and SCUDI, J. V. (1948), Proc. Soc. Exp. Biol. Med., 67, 159.

ERDEI, A. (1948), Lancet, $1,791$.

ERDEI, A. (1948b), Lancet 2, 118.

FASHENA, G. J., and WALKER, J. N. (1944), Amer. F. Dis. Child., 68, 369.

FELDMAN, W. H., KARLSON, A. G., and HINSHAW, H. (1947), Proc. Staff Meetings, Mayo Clinic, 22, 473.

HONIGSBERGER, M.,(1943), Brit. Med. F., 2, 57.

HURST, A., and LINTOTT, G. H. M. (1939), Guy's Hosp. Rep., 89, 173.

HURST, A. (1943), Brit. Med. F., r, 768.

KEITH, J. D., and HICKMANS, E. M. (1938), Arch. Dis. Child., 13, 135.

KONDO and NAKAJIMA (1922), F. Pharm. Soc., Fapan, 485, 355. LEHMAN, J. (1946a), Lancet, 1, 14.

LEHMAN, J. (1946b), Lancet, 1, 15.

LEHMAN, J. (1946c)," Svenska Lakarlidningen., 43, 2029.

LINDSAY, L. M. (1937), Amer. F. Dis. Child., 54, 952. 
LINK, K. P., and others (1943), F. Biol. Chem., 147, 463.

MCCLOSKY, W. T., SMITH, M. I., and FRIAS, J. E. G. (1948), F. Pharmacol. Exp. Therapeutics, 92, 447.

MAYER, O. O., and HOWARD, B. (1943), Proc. Soc. Exp. Biol. Med., 53, 234.

O'CONNOR, J. A. (1948a), Lancet, I, I9r.

O'CONNOR, J. A. (1948b), Proc. Roy. Soc. Med., 6r, 3 ro.

PELNER, F. (1942), J. Lab. Clin. Med., 28, 28.

RAGAZ, L. (1948), Schweiz. med. Wchnochr., 78, 332.

ROSDAHL, K. G. (1948a), Svensk. Kemisk, Tidskrift, 60, 12.

ROSDAHL, K. G. (1948b), Svensk. Kemisk. Tidskrift (in English), $60,64$.

SHAPIRO, S. (1944), 7. Amer. Med. As., 125, 546.

SEYMOUR, D. E., and MCANALLY, D. (1948), Lancet, I, 303.

SEYMOUR, D. E. (1948), Personal communication.

SIEVERS, O. (1946)," Svenska Lakartidningen, 43, 2041.

SPRING, F. S., MARTIN, D. D., and others (1948), Nature, 161, 435.

TENNANT, D. M., and LELAND, M. L. (1948), Federation Proceedings, 7, 195.
TROLL, M. M., and MENTEN, M. L. (1945), Amer. f. Dis. Child., 69, 37 .

VALLENTIN, G. (1946)* Svenska Lakartidningen, 43, 2047.

VENKATARAMAN, A., and others (1948), f. Biol. Chem., 173, 641.

WAY, E. L., WEISS, R., and others (1948), Federation Proceedings, 7,263 .

WHITTET, T. D. (1947), Pharm. F., $133,105$.

WHITTET, T. D. (1948), Lancet, I, 268.

YOUMANS, G. P. (1946), Quart. Bull. North West. Univ. Med. School, 20, 420.

YOUMANS, G. P., RALEIGH, G. W., and YOUMANS, A. (1947), F. Bact., 54, 409.

- Some confurion has arisen in the citation of papers by Lehman (1946c), Sievers (1946) and Vallentin (1946). These originally appeared in Svenska Lakartidningen as quoted here, but were reprinted the following year (1947) in Nordisk Medicin. It is the reprinted papers alone that are cited in the Cumulative Index Medicus.

\section{LAY CLINICIANS}

No. 14

Often when reading a book or play one comes across an excellent description of some disease. Such accounts are always of interest to the doctor and they often show surprisingly accurate powers of observation and clinical acumen. We hope to print some of these 'case records' from time to time and for those who would like to try their hand at naming the author, this information will be given at the end of the extract.

'When I knew her she was not only pitiable but hideous. I do not know what her illness was, but her face was swollen as if it had been stung by bees. Her eyes were just narrow slits between two swollen shiny cushions without eyebrows. Similarly swollen, shiny, and yellow were her cheeks, nose, lips, and mouth. She spoke with difficulty (having probably a similar swelling in her mouth). In summer, flies used to settle ons her face and she did not feel them, which was particularly unpleasant to witness. Her hair was - black but scanty, and did not hide her scalp. . . . A bad smell always came from her, and in her little room, the windows of which were never opened, the odour was stifling.'

This account of myxoedema is taken from Leo Tolstoy's 'Recollections and Essays.' Tolstoy, who is perhaps best known for his two great novels, 'War and Peace' and 'Anna Karenina,' was a most careful observer of his fellow men.

\section{RUTHIN CASTLE, NORTH WALES}

A Clinic for the diagnosis and treatment of Internal Diseases (except Mental or Infectious Diseases). The Clinic is provided with a staff of doctors, technicians and nurses.

The surroundings are beautiful. The climate is mild. There is central heating throughoút. The annual rainfall is 30.5 inches, that is, less than the average for England.

The Fees are inclusive and vary according to the room occupied.

For particulars apply to THE SECRETARY, Ruthin Castle, North Wales.

Tolegrams : Castle, Ruthin.

Telephone: Ruchin 66 